\title{
AC voltage regulating system based on full-bridge inverter Wei Qi ${ }^{*}$, Liangchun Wang, Qiuting Wang, Jian Fang, Duo Xiao
}

School of information and electrical engineering, Zhejiang University City College, Hangzhou, 310015, China

Keywords: voltage regulator, bipolar SPWM, inverter.

\begin{abstract}
A low cost indirect type AC voltage regulating system is proposed to overcome the grid pollution of silicon controlled regulating circuit and the high cost of chopping sine wave regulating circuits. The system consists of full bridge rectifier, filter and full bridge inverter. The bipolar SPWM control signal for inverter is generated by the STM32 micro controller. The circuits is developed and used in the dimming of $400 \mathrm{~W}$ high pressure sodium lamp inductive loads. Continuous dimming with slight distortion of the sine wave is obtained. The system is attractive in the replacement of silicon controlled dimming circuit, and promotes the energy saving project.
\end{abstract}

\section{Introduction}

China's urbanization continues to accelerate, owning to people's greatly improved living standard and the development of the economy. As an important indicator of urban modernization, traffic lighting and landscape lighting is developing rapidly and consuming more and more energy. Energy shortage and environmental pollution problems is a common concern of all humanity, energy and environmental protection are increasingly being taken seriously. According to the external light intensity and flow of people, appropriate steps down lightening, can effectively save energy, thus reduce carbon emissions [1]. The AC regulating system is the core block for light dimming. The AC regulating circuit based on Silicon Controlled Rectifier (SCR) is used widely in dimming [2], with advantages of low cost and simple structure. However, the SCR based dimming circuit adjusts the output voltage by changing the conduction angle of SCR. Thus the output is not a standard sine wave, but contains a large number of high-order harmonics, which will disturb other equipment's in the grid. With the development of electronic devices IGBT, dimming circuit based on chopping is proposed and used in the stage dimming, as well as hotels [3]. The output is pure sine wave and will not affect other equipment's in the grid any more. However, the chopping based dimming circuit is relatively complex and the filter circuit depends on the load characteristics, which resulting in higher system costs and can only be applied in expensive entertainment places [4].

In this paper, a low cost AC regulating system with simple structure is proposed and realized. The output is continuously adjustable standard sine wave, and compatible for resistive, inductive and capacitive loads.

\section{System Design}

The system consists of full bridge rectifier, full-bridge inverter and filer circuit, as shown in Fig. 1. The input $220 \mathrm{~V} / 50 \mathrm{HZ}$ sine wave is rectified to DC voltage firstly, and then enters into the fullbridge inverter circuit and filter circuit, where the input DC voltage is inverted to AC voltage. The output voltage is adjusted by changing the control signal of full-bridge inverter. The output is insensitive with the load characteristics, owning to the inverter circuit. 


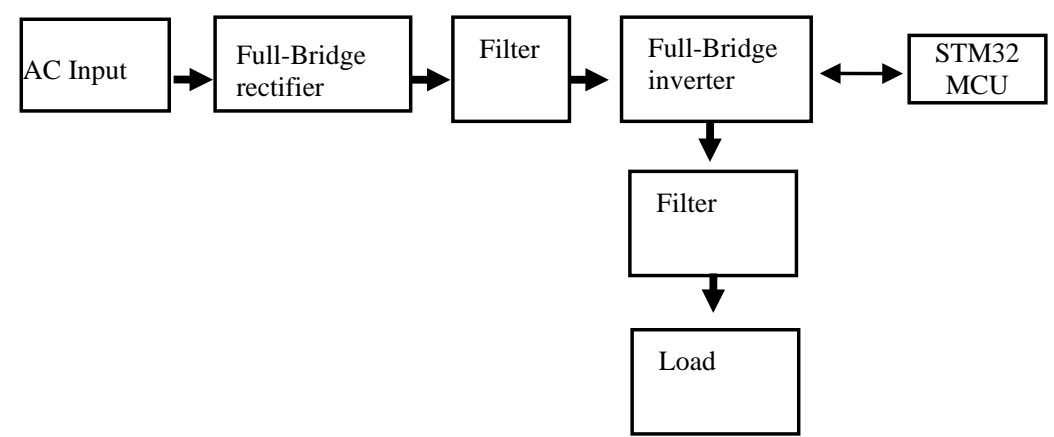

Fig. 1: Schematic of the indirect regulating system

The full-bridge inverter is the core block of the system. The inverter consists of 4 series- and parallel -connected IGBT devices, as shown in Fig. 2. The 4 IGBTs are driven by SPWM signal generated by the STM32 microcontroller. According to detected voltage and current of the input and output ports, the STM32 microcontroller dynamically changes the SPWM driving signal to satisfy the output voltage. These are two types of SPWM driving signal, unipolar SPWM and bipolar SPWM. For inductive load in our application, bipolar SPWM is chosen for driving the IGBTs, since it is far easier to get a good output waveform. In Fig. 2, the left half-bridge consists of IGBT $\mathrm{T}_{1}$ and $\mathrm{T}$, and the right half-bridge consists of IGBT $\mathrm{T}_{3}$ and $\mathrm{T}_{4}$. For bipolar IGBT driving circuit, the high-side and low-side IGBTs (e.g. $\mathrm{T}_{1}$ and $\mathrm{T}_{2}$ ) have complementary driving signal, and the diagonal IGBTs (e.g. $\mathrm{T}_{1}$ and $\mathrm{T}_{4}$ ) have the same driving signal. The output is close the peak of the sine signal as the duty cycle of the SPWM is close to $0 \%$ or $100 \%$, meanwhile the output is close to 0 as the duty cycle of the SPWM is close to 50\%, which is shown in Fig. 3. To simplify the system complexity and lower the cost, two complementary driving signals with dead zone are generated by the STM32.

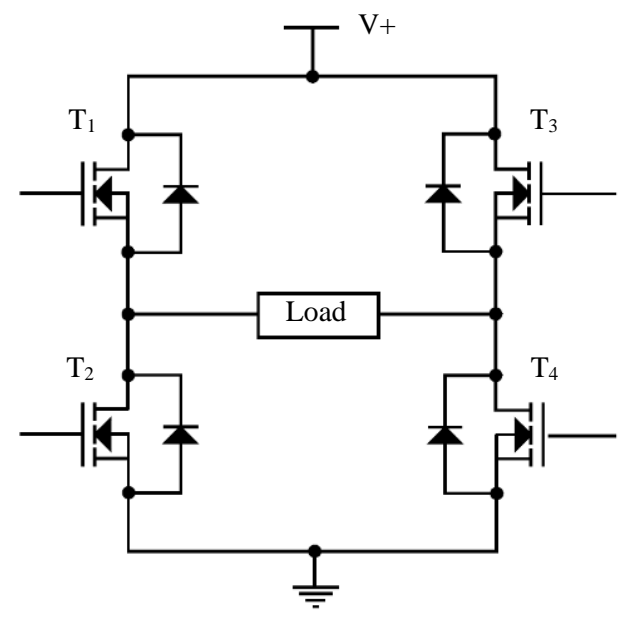

Fig. 2: Topology of full-bridge inverter

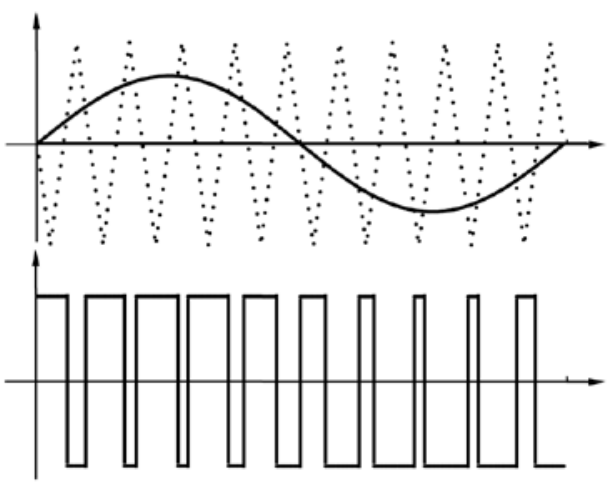

Fig. 3: Bipolar SPWM driving signal 


\section{Circuit Design}

Power circuit.

The power circuit consists of rectifier, full-bridge inverter and output filter circuit. To lower the system cost, simple full-bridge rectifier and filter capacitor are used for rectifier. As shown in Fig. 4, the input 220V AC is converted to DC after the rectifier. The full-bridge inverter consists of two half-bridge, as shown in Fig. 5. The high-side and low-side IGBTs are driven by complementary signals with dead zone, to avoid the simultaneous conducting.

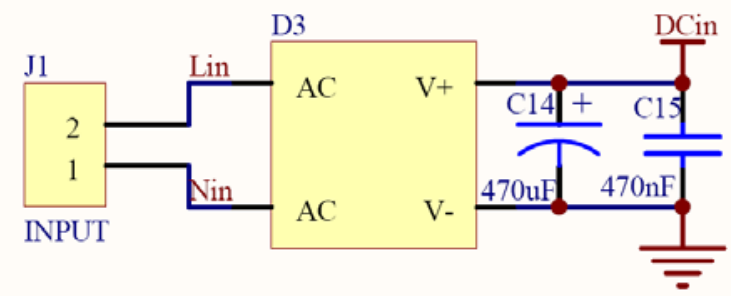

Fig. 4: Full-bridge rectifier and filter

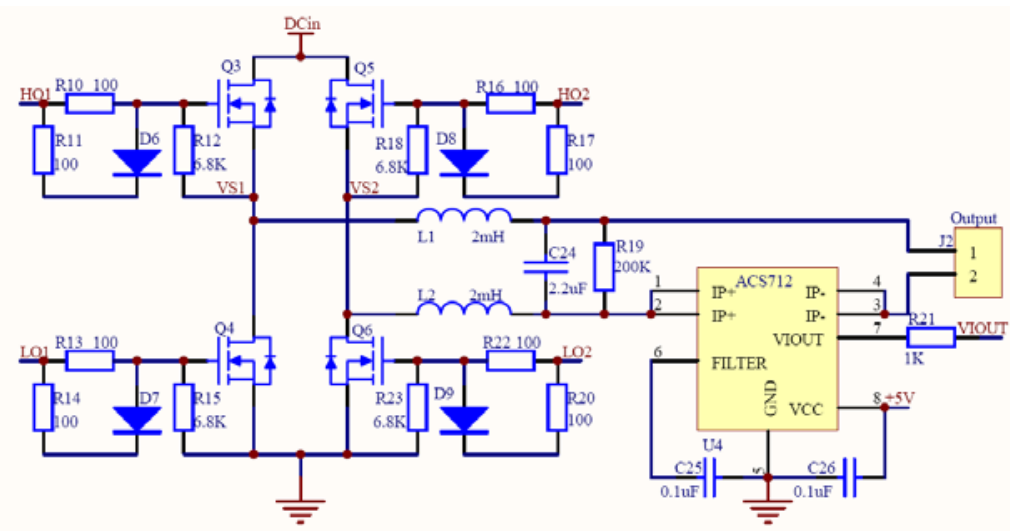

Fig. 5: Full-bridge inverter

\section{IGBT driving circuit.}

STM32 MCU contains enhanced PWM module, the module can generate a PWM signal on up to different output pins with up to 10bits of resolution, and it can do this through for different PWM output modes. In our application, the full-bridge is two half-bridge actually, thus the module is configured to Half-Bridge PWM mode. Two complementary signals with dead zone generated by pins $\mathrm{P} 1 \mathrm{~A}$ and $\mathrm{P} 1 \mathrm{~B}$ drive $\mathrm{T}_{1}$ and $\mathrm{T}_{2}$, respectively. The same signals drive $\mathrm{T}_{4}$ and $\mathrm{T}_{3}$ at the same time. The IR2113S devices are used in the IGBT driver design [5]. The gate charge for the high-side IGBT is provided by the bootstrap capacitor which is charged by the $15 \mathrm{~V}$ supply through the bootstrap diode during the time when the device is off (assuming that VS swings to ground during that time, as it does in most applications). Only one power supply is need for driving the high-side and low-side IGBTs, which reduces the number of the power supply in the system. As shown in Fig. 6, two IR2113 are used for driving the two half-bridge. Take the left half-bridge for example, when the high-side IGBT is off and the low-side IGBT is on, the bootstrap capacitor $\mathrm{C}_{13}$ is charged through diode $\mathrm{D}_{4}, \mathrm{C}_{16}$ provides the charge for the high-side IGBT. For reducing the cost, the combination of aluminium electrolytic capacitor $(50 \mu \mathrm{F})$ and ceramic capacitor $(0.1 \mu \mathrm{F})$ is used as bootstrap capacitor. 


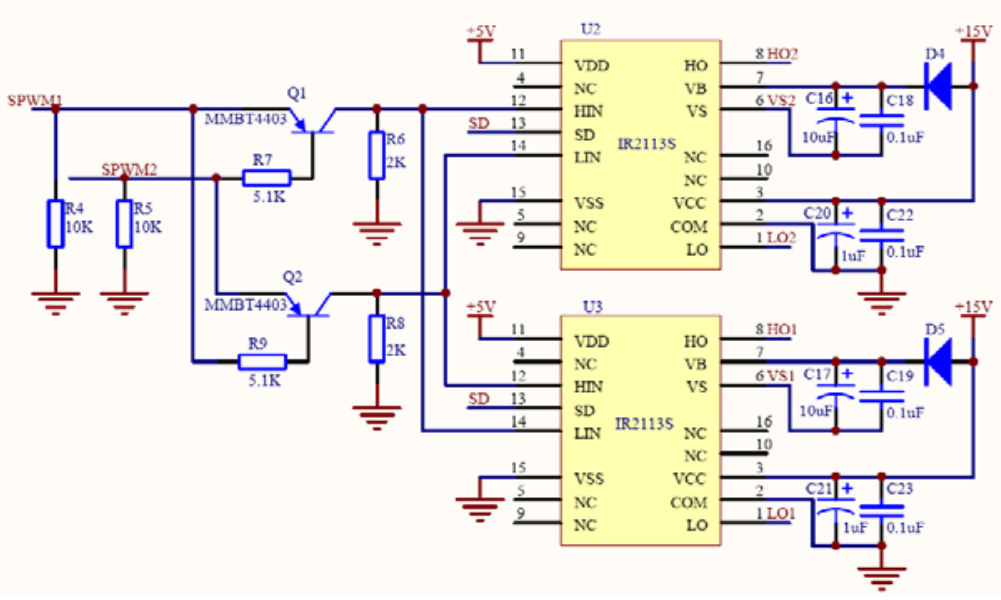

Fig. 6: Full-bridge driving circuit based on IR2113

\section{Generating of SPWM}

The SPWM signal is generated by the STM32 microcontroller, as shown in Fig. 7. The frequency of the grid is $50 \mathrm{~Hz}$, and the PWM frequency is $20 \mathrm{kHz}$ in our design. That is, the period of grid is 400 times that of the PWM. The sine signal is symmetrical waveform, so we only construct the sine table of 1/4 cycle, remain 3/4 cycle can be generated accordingly.

Regular sampling formula of SPWM is defined as:

$$
x_{K}=\sin (K * 2 \pi / N)
$$

Where $\mathrm{K}$ represents the Kth sampling in a fundamental cycle, $\mathrm{N}$ is carrier wave ratio. To lower the complexity, the duty cycle changes every 4 PWM period. Set $\mathrm{K}=4 \mathrm{i}$ and $\mathrm{N}$ :

$$
\begin{aligned}
x_{i} & =\sin (4 i * 2 \pi / 400) \\
& =\sin (i * \pi / 50)
\end{aligned}
$$

Where $i=0 . .24$, the calculated sine table of length 25 is stored in the ROM of STM32.

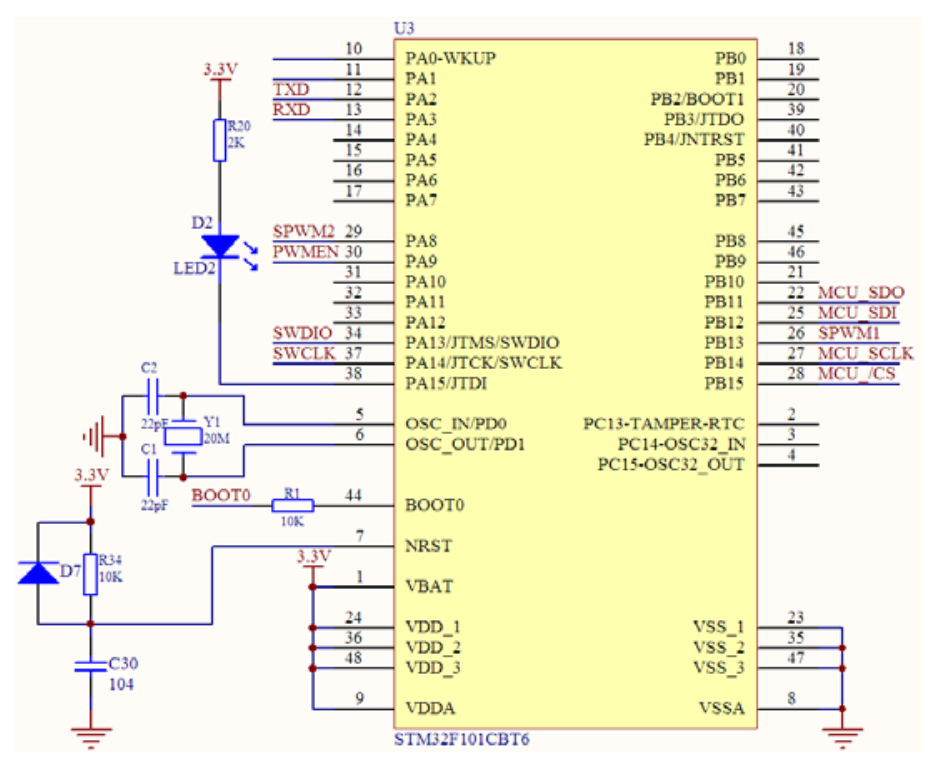

Fig. 7: SPWM generating circuit based on STM32 


\section{Measurement result}

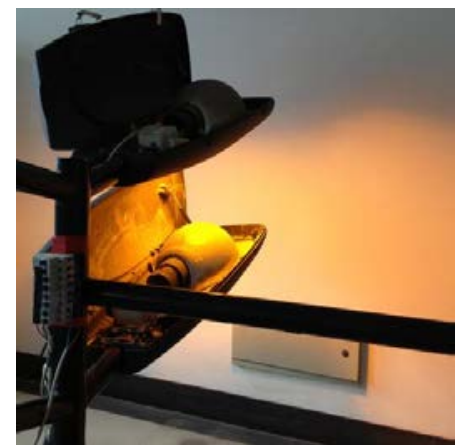

(a)

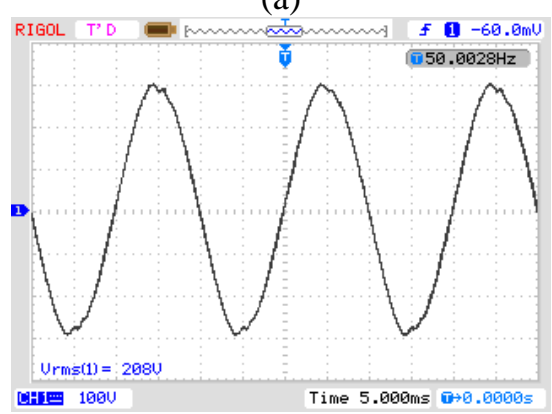

(b)

Fig. 8: Dimming by the regulating system, the output voltage is $208 \mathrm{~V}$

The dead zone is set to $1.6 \mu \mathrm{s}$. The regulating system is series connected between the grid and the high pressure sodium lamp (HPSL). The HPSLs are used widely in street lighting, which serve as conductive load in the measurement. Fig. 8 shows the dimming state with output voltage of 208V, the output wave has slight distortion compared with standard sine wave. When the output voltage is adjusted to $162 \mathrm{~V}$, the output wave is similar to standard sine wave, as shown in Fig. 9.

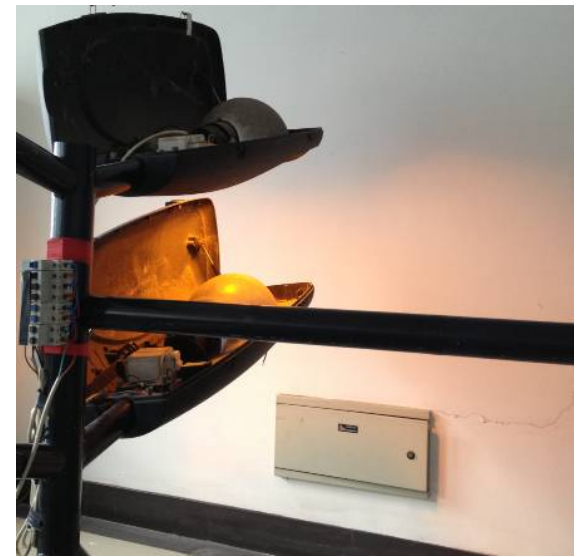

(a)

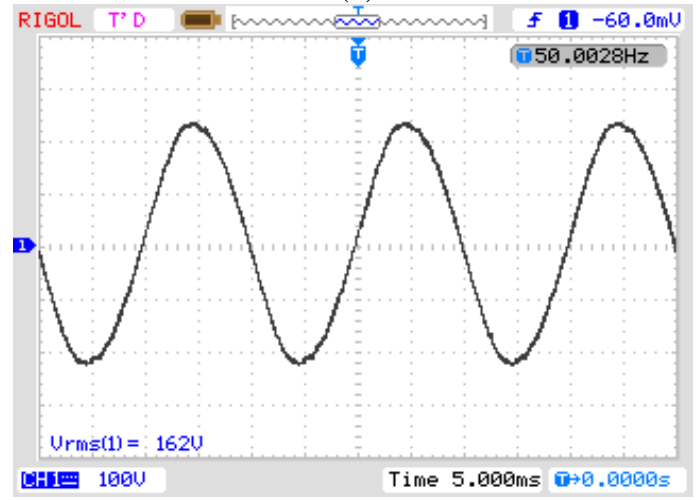

(b)

Fig. 9: Dimming by the regulating system, the output voltage is $162 \mathrm{~V}$ 


\section{Conclusions}

An indirect regulating system based on AC/DC/AC is proposed and realized, the system successfully dims the high pressure sodium lamp, and the output wave has only slight distortion. The regulating system has simple structure, low cost and no pollution to the grid, and is expected to replace the existing SCR based dimming system.

\section{Acknowledgements}

This work was supported by the Science and Technology Department of Zhejiang Province (No. 2014C33026), Zhejiang University City College Scientific Research Foundation (No. J-14022), Hangzhou Municipal Science and Technology Project (No. 20130533B26), the construct program of the top priority discipline in Hangzhou and the construct program of the top priority laboratory in Hangzhou.

\section{References}

[1] Lin, Z. G., The Energy saving Application of High Pressure Sodium lamp in Urban Road Lighting. China Illuminating Engineering Journal, 20(4), p. 74-77, 2009. (In Chinese)

[2] Wu, S. L., Design of NC/AC Voltage Regulator Based on STCl2C5410AD SCM. Manufacture Information Engineering of China, 38(11), p. 54-60, 2009. (In Chinese).

[3] Yu, X. W., Zou, Y. P. , Zhang, J. \& Lu, L. , Application of AC Voltage Regulator with ChopControl in Sine-Wave Regulator Equipment's. Telecom Power Technologies, 24(2), p. 43-48, 2007. (In Chinese).

[4] Su, X. Y., Chen, B. D., Ding, H. Y. \& Zhang, X. Y., Design of AC Chopper Regulator to Control the Voltage Based on DSP. Journal of Heilongjiang Hydraulic Engineering, 36(1), p. 89-91, 2009. (In Chinese).

[5] Zhang, M. \& Zhang, B. G. Improvement design of IR2110 drive circuit. Electronic Design Engineering, 17(12), p. 66-67, 2009. (In Chinese). 